\title{
Synthesis of new disulfonamides from different substituted diamino pyridines
}

\author{
Hossein Behmadi ${ }^{a,}$, Seyed Mahdi Saadati ${ }^{a}$,Mina Roshani ${ }^{a}$, Mousa Ghaemy ${ }^{b}$ \\ Department of Chemistry, Faculty of Basic Sciences, Islamic Azad University, Mashhad Branch, Mashhad 91735-413, Iran ${ }^{a}$ \\ Faculty of Chemistry, University of Mazandaran, Babolsar 47416-1467, Iran ${ }^{b}$ \\ *hos_behmadi@yahoo.com; behmadi@mshdiau.ac.ir
}

\begin{abstract}
A series of novel disulfonamide derivatives were synthesized and characterized by FT-IR, ${ }^{1} \mathrm{H}$ NMR and MS techniques. In order to prepare new disulfonamides, at first we synthesized new diamines containing a pyridine ring. Then, they have been reacted with sulfonyl chlorides to give corresponding disulfonamides.
\end{abstract}

Keywords: Sulfonamides, Triaryl pyridine, Diamino pyridine, Disulfonamides.

\section{Introduction}

Sulfonamides are a very important class of compounds in the pharmaceutical industry, being widely used as anticancer, anti-inflammatory and antiviral agents [1]. Over 30 drugs containing this functionality are in clinical use, including, antibacterials, diuretics, anticonvulsants, hypoglycemics and HIV protease inhibitors [2]. More recently, sulfonamides have been found to be potent cysteine protease inhibitors, which could possibly extend their therapeutic applications to include conditions such as Alzheimer's disease, arthritis and cancer [3]. As a class; the sulfa drugs have a veritable history of application for the treatment of bacterial infection [4]. Sulfonamides are among the most widely used antibacterial agents in the world, chiefly Because of their low cost, low toxicity and excellent activity against common bacterial diseases [5].

Arylsulfonyl substituents have been used as protecting groups for oxygen and nitrogen functionalities [6]. Sulfonamide derivatives of azo dyes achieve improved light stability, water solubility, and fixation to fiber [7]. Typically, sulfonamides were prepared by the reaction of a sulfonyl chlori- de with ammonia or primary or secondary amines [8]. Caddick and co-workers reported a suitable preparation of sulfonamides by intermolecular radical addition to pentafluorophenyl vinylsulfonate and successive aminolysis [9]. Katritzky and co-workers proposed a general and efficient synthesis of sulfonamides by the reaction between sulfonylbenzotriazoles (produced from sulfinic acid salts with $N$-chlorobenzotriazole) and various amines [10]. Recently the synthesis of heteroaryl sulfonamides via oxidation of thiols to sulfonyl chlorides or sulfonyl fluorides has been reported that were then reacted with amines to give the corresponding sulfonamides [11]. The logical way to sulfonamides could be the direct synthesis from sulfonic acid. Even if pharmaceutical compounds containing a sulfonamide group have numerous significant therapeutic applications, at present just two methodologies are reported to convert a sulfonic acid directly to a sulfonamide. The first method permits the synthesis of sulfonamides from the sulfonic acid pyridine or triethylamine salts by the use of the activating agent triphenylphosphine ditriflate [12]. The second procedure considers the reaction of a sulfonic acid with isocyanides at room temperature [13]. 
Pyridine with a 2,4,6-triaryl substitution pattern (Kröhnke pyridine) [14] have been synthesized using various methods and procedures. Traditionally, these compounds have been synthesized through the reaction of $N$-phenacylpyridinium salts with $\alpha, \beta$-unsaturated ketones in the presence of $\mathrm{NH}_{4} \mathrm{OAc}$ $[14,15]$. However, the pyridinium salts and the unsaturated ketones have to be synthesized first, so this method is relatively expensive. More recently, several new improved methods and procedures have been developed for the synthesis of these pyridines: reaction of $\alpha$-ketoketene dithioacetals with methyl ketones in the presence of $\mathrm{NH}_{4} \mathrm{OAc}$ [16], reaction of $\mathrm{N}$-phosphinylethanimines with aldehydes [17], addition of lithiated $\beta$-enaminophosphonates to chalcones [18], solvent-free reaction between acetophenones, benzaldehydes, and $\mathrm{NH}_{4} \mathrm{OAc}$ in the presence of sodium hydroxide [19], and the one-pot reaction of acetophenones, benzaldehydes, and $\mathrm{NH}_{4} \mathrm{OAc}$ without catalyst under microwave irradiation [20].

In this paper we report synthesis and structural elucidation of new disulfonamides 5a-f in three steps (Scheme 1).

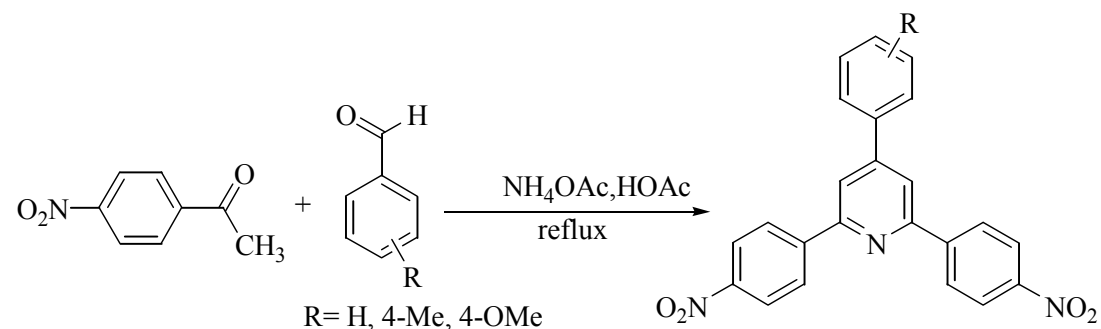

(1)<smiles>[R][X]c1ccc(-c2cc(-c3ccc([N+](=O)[O-])cc3)nc(-c3ccc([N+](=O)[O-])cc3)c2)cc1</smiles>

$(3 a-c)$<smiles>[R]c1ccc(-c2cc(-c3ccc(N)cc3)nc(-c3ccc(N)cc3)c2)cc1</smiles>

(4a-c)
(3)

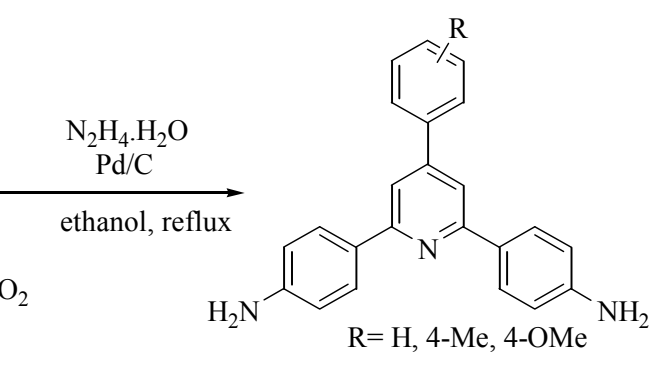

(4a-c)<smiles></smiles>

(5a-f)
$\mathrm{R}=\mathrm{H}, 4-\mathrm{Me}, 4-\mathrm{OMe}$

$\mathrm{R}^{\prime}=4-\mathrm{MeC}_{6} \mathrm{H}_{4},{ }_{4}-\mathrm{ClC}_{6} \mathrm{H}_{4}$

Scheme 1. Synthesis of disulfonamides 


\section{Experimental Section}

All yields refer to isolated products. Melting points were recorded on an electrothermal Stuart SMP3. The IR spectrometer used was Bruker Tensor 27 . The IR spectra were taken using $\mathrm{KBr}$ pellets. The ${ }^{1} \mathrm{H}$ NMR $(100 \mathrm{MHz})$ spectra were recorded on a Bruker AC 100 spectrometer. The mass spectra were scanned on a 5973 Network Mass Selective Detector, Agilent Technology (HP).

\section{Typical procedure for the preparation of compounds 3a-c and 4a-c}

\section{4-phenyl-2,6-bis(4-nitrophenyl) pyridine 3a}

This compound was synthesized according to the following procedure [21]. In a round-bottomed flask $(250 \mathrm{~mL})$ equipped with a reflux condenser, a mixture of benzaldehyde $(6.4 \mathrm{~g}, 0.06 \mathrm{~mol})$, p-nitroacetophenone (20 g, $0.12 \mathrm{~mol})$, ammonium acetate $(60 \mathrm{~g})$, and glacial acetic acid $(150 \mathrm{ml})$ was refluxed for $2 \mathrm{~h}$. Upon cooling, crystals separated, which were filtered and washed first with acetic acid $(50 \%)$ and then with cold ethanol. These dark yellow crystals were recrystallized from absolute ethanol, and then dried at $60{ }^{\circ} \mathrm{C}$ under vacuum. The product was 4-phenyl-2,6-bis(4-nitrophenyl) pyridine 3a. The other dinitropyridines $\mathbf{3 b}, \mathbf{3 c}$ were synthesized in the same procedure.

\section{4-Phenyl-2, 6-bis (4-aminrophenyl) pyri- dine 4a}

In a two-necked round-bottomed flask (1000 ml) equipped with a reflux condenser and a dropping funnel, a suspension of 4-phenyl-2,6bis(4-nitrophenyl) pyridine 3a (13.75 g, 0.033 $\mathrm{mol})$, palladium on carbon $5 \%(1.4 \mathrm{~g})$, and ethanol $(500 \mathrm{~mL})$ was prepared. The mixture was warmed, and while being stirred magnetically, hydrazine hydrate $85 \%(35 \mathrm{ml})$ in ethanol $(50 \mathrm{ml})$ was added dropwise over a $1.5 \mathrm{~h}$ period through the dropping funnel while maintaining the temperature at about $50{ }^{\circ} \mathrm{C}$. The reaction mixture was then refluxed for $2 \mathrm{~h}$ and filtered while hot. On cooling, the filtrate gave white-cream colored crystals of the title diamine compound, which were recrystallized from ethanol and vacuum dried. The other diaminopyridines $\mathbf{4 b}, \mathbf{4 c}$ were synthesized in the same procedure.

\section{Typical procedure for the preparation of disulfonamides 5a-f}

The nucleophilic substitution reaction of the diamines 4a-c with 4-methylbenzene-1sulfonyl chloride or 4-chlorobenzene-1-sulfonyl chloride was carried out as follows: to the THF solution of diamines $(1 \mathrm{mmol})$ and triethyl amine $(2 \mathrm{mmol})$ was added by slowly dropwise THF solution of arylsulfonyl chloride $(2.2 \mathrm{mmol})$ while the temperature maintain between 0 and $-5{ }^{\circ} \mathrm{C}$. Then, the reaction mixture was stirred at room temperature. The progress of the reaction was monitored by TLC (eluent: n-hexane / EtOAc: 3/2). After the completion of the reaction, solvent was evaporated in vaccum. At least the solid residue was recrystallized from ethanol.

\section{Results and Discussion}

The structures of the products were deduced from their spectral data. For example, the ${ }^{1} \mathrm{HNMR}$ spectrum of $\mathbf{5 c}$ in $\mathrm{d}_{6}$-DMSO shows a singlet at $\delta=2.31\left(6 \mathrm{H}, 2-\mathrm{CH}_{3}\right)$, a singlet at $\delta=3.82(3 \mathrm{H}$, $\left.-\mathrm{OCH}_{3}\right)$, a multiplet at $\delta=7.1-8.1(22 \mathrm{H}, \mathrm{ArH})$, and a singlet at $\delta=10.3-10.5\left(2 \mathrm{H}, 2 \mathrm{NH}, \mathrm{D}_{2} \mathrm{O}\right.$-exchangeable) ppm. Mass spectrum showed the expected molecular ion peak and fragmentation pattern is according with the proposed structure.

The IR spectra of 5a-f show three bands at $3034-3089 \mathrm{~cm}^{-1}, 1340 \mathrm{~cm}^{-1}$ and $1160 \mathrm{~cm}^{-1}$ indicative of $\mathrm{NH}$ and $\mathrm{SO}_{2}$ groups.

In conclusion, we have synthesized new disulfonamides 5a-f containing pyridine ring without using any catalyst. The reaction was carried out under easy conditions and is high yielding and product isolation is very straightforward. Several derivatives of the title compounds with different substituents were synthesized to show the diversity of the method. 


\section{Spectral data}

4-methyl-N $N^{1}-\{4-[6-(4-\{[(4-m e t h y l p h e n y l)$ sulfonyl]amino ? phenyl-4-phenyl-2-pyridyl] phenyl\}-1-benzenesulfonamide $\mathbf{5 a}$

Orange powder, Yield: $80 \%, \mathrm{mp} 223-225{ }^{\circ} \mathrm{C}$; ${ }^{1} \mathrm{H}$ NMR (100 MHz, d $\left.-\mathrm{DMSO}\right): \delta=2.26(\mathrm{~S}$, $\left.2 \mathrm{CH}_{3}\right), 7.1-8.5(\mathrm{~m}, 23 \mathrm{H}, \mathrm{ArH}), 10.4-10.6$ (S, $2 \mathrm{NH}, \mathrm{D}_{2} \mathrm{O}$-exchangeable) ppm; IR: $\mathrm{v}=3089$ $(\mathrm{NH}), 1332(\mathrm{~S}=\mathrm{O}), 1160(\mathrm{~S}=\mathrm{O}) \mathrm{cm}^{-1} ; \mathrm{MS}$ $(70 \mathrm{eV}): m / z=645\left(\mathrm{M}^{+}\right)$.

4-methyl-N $N^{1}-\{4-[6-(4-\{[(4-m e t h y l p h e n y l)$ sulfonyl]amino? phenyl-4-(4-methylphenyl)-2pyridyl]phenyl\}-1-benzenesulfonamide $\mathbf{5 b}$

Orange powder, Yield: $86 \%, \mathrm{mp} 208-210{ }^{\circ} \mathrm{C}$; ${ }^{1} \mathrm{H}$ NMR (100 MHz, d ${ }_{6}$ DMSO): $\delta=2.36(\mathrm{~S}$, $\left.3 \mathrm{CH}_{3}\right), 7.2-8.4(\mathrm{~m}, 22 \mathrm{H}, \mathrm{ArH}), 10.3-10.5(\mathrm{~S}$, 2NH, $\mathrm{D}_{2} \mathrm{O}$-exchangeable) ppm; IR: $v=3034$ $(\mathrm{NH}), 1332(\mathrm{~S}=\mathrm{O}), 1159(\mathrm{~S}=\mathrm{O}) \mathrm{cm}^{-1} ; \mathrm{MS}$ (70eV): $m / z=659\left(\mathrm{M}^{+}\right)$.

4-methyl-N $N^{1}-\{4-[6-(4-\{[(4-m e t h y l p h e n y l)$ sulfonyl]amino ? phenyl-4-(4-methoxyphenyl)-2pyridyl]phenyl\}-1-benzenesulfonamide $\mathbf{5 c}$

Yellow powder, Yield: $90 \%, \mathrm{mp} 245-248{ }^{\circ} \mathrm{C}$; ${ }^{1} \mathrm{H}$ NMR (100 MHz, d $\left.-\mathrm{DMSO}\right): \delta=2.31(\mathrm{~S}$, $\left.2 \mathrm{CH}_{3}\right), 3.82\left(\mathrm{~S},-\mathrm{OCH}_{3}\right), 7.1-8.1(\mathrm{~m}, 22 \mathrm{H}$, ArH), 10.3-10.5 (S, 2NH, $\mathrm{D}_{2} \mathrm{O}$-exchangeable) ppm; IR: $v=3061(\mathrm{NH}), 1341(\mathrm{~S}=\mathrm{O}), 1161$ $(\mathrm{S}=\mathrm{O}) \mathrm{cm}^{-1} ; \mathrm{MS}(70 \mathrm{eV}): \mathrm{m} / z=675\left(\mathrm{M}^{+}\right)$.

4-chloro- $N^{1}-\{4-[6-(4-\{[(4-c h l o r o p h e n y l)$ sulfonyl]amino? phenyl-4-phenyl-2-pyridyl] phenyl\}-1-benzenesulfonamide $\mathbf{5 d}$

Red powder, Yield: $85 \%$, mp $236{ }^{\circ} \mathrm{C}$; ${ }^{1} \mathrm{H}$ NMR (100 MHz, d d $\left.^{-D M S O}\right): \delta=7.2-8.2(\mathrm{~m}, 23 \mathrm{H}$, ArH), 10.4-10.6 (S, 2NH, $\mathrm{D}_{2} \mathrm{O}$-exchangeable) ppm; IR: $v=3059(\mathrm{NH}), 1337(\mathrm{~S}=\mathrm{O}), 1162$ $(\mathrm{S}=\mathrm{O}) \mathrm{cm}^{-1}$; MS $(70 \mathrm{eV}): m / z=685\left(\mathrm{M}^{+}\right)$.

4-chloro- $N^{1}-\{4-[6-(4-\{[(4-c h l o r o p h e n y l) s u l-$ fonyl]amino phenyl-4-(4-methylphenyl)-2pyridyl]phenyl\}-1-benzenesulfonamide $\mathbf{5 e}$

Brown powder, Yield: 88\%, mp 205-208 ${ }^{\circ} \mathrm{C} ;{ }^{1} \mathrm{H}$ NMR (100 MHz, d $\mathrm{d}_{6}$ DMSO): $\delta=2.37\left(\mathrm{~S}, \mathrm{CH}_{3}\right)$, 7.2-8.4 (m, $22 \mathrm{H}, \mathrm{ArH}), 10.4-10.6(\mathrm{~S}, 2 \mathrm{NH}$, $\mathrm{D}_{2} \mathrm{O}$-exchangeable) ppm; IR: $v=3088(\mathrm{NH})$,
$1335(\mathrm{~S}=\mathrm{O}), 1162(\mathrm{~S}=\mathrm{O}) \mathrm{cm}^{-1} ; \mathrm{MS}(70 \mathrm{eV}): \mathrm{m} / \mathrm{z}$ $=699\left(\mathrm{M}^{+}\right)$.

4-chloro- $N^{1}-\{4-[6-(4-\{[(4-c h l o r o p h e n y l)$ sulfonyl]amino\} phenyl-4-(4-methoxyphenyl)-2pyridyl]phenyl\}-1-benzenesulfonamide $\mathbf{5 f}$

Yellow powder, Yield: 92\%, mp $260-262{ }^{\circ} \mathrm{C}$; ${ }^{1} \mathrm{H}$ NMR (100 MHz, d 6 -DMSO): $\delta=3.81(\mathrm{~S}$, $\left.\mathrm{CH}_{3}\right), 7.1-8.3(\mathrm{~m}, 22 \mathrm{H}, \mathrm{ArH}), 10.4-10.6(\mathrm{~S}$, 2NH, $\mathrm{D}_{2} \mathrm{O}$-exchangeable) ppm; IR: $v=3076$ $(\mathrm{NH}), 1345(\mathrm{~S}=\mathrm{O}), 1163(\mathrm{~S}=\mathrm{O}) \mathrm{cm}^{-1} ; \mathrm{MS}$ $(70 \mathrm{eV}): m / z=717(\mathrm{M}+1)$

\section{References:}

[1] (a) C. T. Supuran, A. Casini and A. Scozzafava, Med. Res. Rev., 5 (2003) 535.

(b) A. Scozzafava, T. Owa, A. Mastrolorenzo and C. T. Supuran, Curr. Med. Chem., 10 (2003) 925.

[2] A. Kleemann, J. Engel, B. Kutscher, D. Reichert, Eds; Pharmaceutical Substances, Syntheses, Patents, Applications, (Thieme, Stuttgart, 1999).

[3] (a) W. R. Roush, S. L. Gwaltney, J. Cheng, K. A. Scheidt, J. H. McKerrow, E. Hansell, J. Am. Chem. Soc., 120 (1998) 10994.

(b) W. R. Roush, J.Cheng, B. Knapp- Reed, A. AlvarezHernandez, J. H. McKerrow, E. Hansell, J. C. Engel, Bioorg. Med. Chem. Lett., 11 (2001) 2759.

[4] R. Pandya, T. Murashima, L. Tedeschi, A. G. M. Barrett, J. Org. Chem., 68 (2003) 8274.

[5] N. Özbek, H. Katırcioğlu, N. Karacan, T. Baykal, Bioorg. Med. Chem., 15 (2007) 5105.

[6] J. F. O'Connel, H. Rapoport, J. Org. Chem., 57 (1992) 4775.

[7] C. Hansch, P. G. Sammes, J. B. Taylor, Comprehensive Medicinal Chemistry, (Pergamon Press, Oxford, 1990) Vol. 2, Chapter 7.1.

[8] L. De Luca, G. Giacomelli, J. Org. Chem., 73 (2008) 3967.

[9] S. Caddick, J. D. Wilden, H. D. Bush, S. N. Wadman, D. N. Judd, Org. Lett., 4 (2002) 2549.

[10] A. R. Katritzky, V. Rodriguez-Garcia, S. K. Nair, J. Org. Chem., 69 (2004) 1849.

[11] S. W. Wright, K. N. Hallstrom, J. Org. Chem., 71 (2006) 1080.

[12] S. Caddick, J. D. Wilden, D. B. Judd, J. Am. Chem. Soc., 126 (2004) 1024.

[13] A. Shaabani, E. Soleimani, A. H. Rezayan, Tetrahedron Lett., 48 (2007) 2185.

[14] F. Kröhnke, W. Zecher, Angew. Chem., Int. Ed., 1 (1962) 626 . 
[15] F. Kröhnke, Synthesis, (1976) 1.

[16] (a) K. T. Potts, M. J. Cipullo, P. Ralli, G. Theodoridis, J. Am. Chem. Soc., 103 (1981) 3584.

(b) K. T. Potts, M. J. Cipullo, P. Ralli, G. Theodoridis, J. Am. Chem. Soc. 103 (1981) 3585.

[17] T. Kobayashi, H. Kakiuchi, H. Kato, Bull. Chem. Soc. Jpn., 64 (1991) 392.

[18] F. Palacios, A. M. O. de Retana, J. Oyarzabal, Tetrahedron Lett., 37 (1996) 4577.

[19] G. W. V. Cave, C. L. Raston, Chem. Commun., (2000) 2199.

[20] S. Tu, T. Li, F. Shi, F. Fang, S. Zhu, X. Wei, Z. Zong, Chem. Lett., 34 (2005) 732.

[21] B. Tamami, H. Yeganeh, Polymer, 42 (2001) 415. 